\title{
Los anticolinesterásicos mejoran el pronóstico neuropsiquiátrico y funcional de pacientes con Alzheimer: Meta-análisis
}

Efficacy of Cholinesterase Inhibitors in the Treatment of Neuropsychiatric Symptoms and Functional Impairment in Alzheimer Disease.A Meta-analysis. Trinh N, Hoblyn J, Mohanty S y col. JAMA 2003 289:210-216.

\section{Objetivo}

Cuantificar el impacto de los inhibidores de colinesterasa sobre el pronostico neuropsiquiátrico y funcional en pacientes con enfermedad de Alzheimer (EA) de grado leve y moderado.

\section{Fuente y selección de datos}

Se buscó en Medline (Enero 1966-Diciembre 2001), resúmenes On-line, PSYCHINFO, BIOSIS y el Registro de estudios controlados Cochrane. Se revisaron artículos en inglés y en otros idiomas, tanto publicados, como no publicados, contactando investigadores y compañías farmacéuticas. Fueron incluidos 29 estudios aleatorizados (paralelos o cruzados) doble ciego, placebo-control de pacientes no institucionalizados con EA de grado leve a moderado tratados por lo menos un mes con anticolinesterásicos. Dieciséis estudios incluyeron mediciones neuropsiquiatricas y 18 , funcionales.

\section{Extracción de datos}

Dos investigadores extractaron métodos, fuentes de sesgos y resultados. Los resultados neuropsiquiátricos se valoraron mediante las escalas: Neuropsychiatric Inventory (NPI, 0-120 puntos) y del Alzheimer Disease Assesment Scale (ADAS-noncognitive, 0-50 puntos). El funcionamiento diario se evaluó con las escalas
Activities of Daily Living (ADL) y Instrumental Activities of Daily Living (IADL) implicando mejoría un menor puntaje.

\section{Resultados principales}

Los anticolinesterásicos mejoraron modestamente las mediciones en las escalas frente al placebo (ver cuadro 1). No hubo diferencias significativas entre los distintos anticolinesterásicos.

\section{Cuadro 1: resultados de las mediciones de las distintas escalas.}

\begin{tabular}{l|c|l}
\hline Escalas & Node Estudios & Anticolinesterásicos vs Placebo (IC 95\%) \\
\hline $\mathrm{NPI}$ & 10 & $-1,72(0,87$ a 2,57) puntos \\
\hline ADAS-noncogn. & 6 & $-0,03(0,00$ a 0,05) puntos \\
\hline ADL & 14 & $-0,1(0,00$ a 0,19) desvíos estándar \\
\hline IADL & 13 & $-0,09(0,001$ a 0,17) desvíos estándar \\
\hline
\end{tabular}

\section{Conclusiones}

Los inhibidores de colinesterasa tienen un impacto beneficioso modesto sobre escalas de pronóstico neuropsiquiátrico y funcional en pacientes con Alzheimer. Estudios futuros deberían analizar la influencia de esta mejoría en pronóstico a largo plazo, calidad de vida, institucionalización y carga del cuidador.

\section{Comentario}

Tal como expresan los autores en su comentario final, existen varios problemas que restringen la interpretación de resultados en este estudio: 1) no todos los pacientes enrolados en los ensayos analizados presentaban trastornos conductuales, y por esto resulta difícil conocer el verdadero efecto de los anticolinesterásicos sobre dichos problemas; 2) muchos pacientes estudiados recibían benzodiacepinas permitidas en distintos protocolos, lo que podría alterar los resultados; 3) el meta-análisis incluyó diferentes anticolinesterásicos, con diferentes esquemas de tratamiento; 4$)$ los ensayos incluidos no consideraron las escalas conductuales y de funcionalidad como objetivos principales de la evaluación generando datos incompletos en la descripción de estos problemas; 5) no se evaluaron los distintos trastornos de comportamiento y habilidades funcionales por separado, lo que limita la interpretación, ya que el impacto de cada trastorno y perdida de habilidades sobre la vida del paciente y cuidador es diferente; 6) la longitud máxima de los trabajos incluidos fue 210 días y algunos solo duraban 42 días, lo que dificulta evaluar el pronóstico funcional, que requiere estudios prolongados. De todos modos y a pesar de estas consideraciones, los resultados del estudio van en la misma dirección que otras publicaciones.

Los inhibidores de la colinesterasa fueron desarrollados para tratar la enfermedad de Alzheimer sobre la base de la hipótesis colinérgica de la enfermedad. Su utilidad clínica principal radica en mejorar la función cognitiva (principalmente la memoria) al aumentar la neurotransmisión colinérgica a nivel hipocampal.
Todavía no resulta claro el mecanismo fisiopatológico de la mejoría comportamental. Una publicación reciente ${ }^{1}$ no incluida en el presente estudio demuestra una mejoria significativa en los síntomas conductuales de pacientes con Alzheimer moderado y severo con donepecilo y otra, con similares resultados, utilizando igual fármaco $^{2}$ concluye que las diferencias comportamentales basales son predictoras de la respuesta al tratamiento. Ambos estudios tienen como ventaja haber analizado cada trastorno conductual por separado, incluir pacientes severos con importantes trastornos conductuales y haber evaluado donepecilo, la droga mas usada en la práctica. Lamentablemente, el presente estudio carece de dichas ventajas ya que sólo un ensayo utilizó donepecilo, otro rivastigmina y el resto, fármacos sin uso clínico actual.

El anticolinesterásico utilizado no es tema menor ya que si bien todos aumentan la neurotransmisión colinérgica, la diferente farmacodinamia tiene implicancias terapéuticas.

Conclusiones del comentario: Este estudio aporta argumentos a favor del uso de anticolinesterásicos para mejorar la cognición en pacientes con Alzheimer aunque tengan trastornos conductuales. Se requieren estudios prospectivos enfocados primariamente en lo conductual, que evalúen drogas aprobadas para uso clínico considerando el efecto individual sobre cada tipo de comportamiento en individuos con demencia, incluyendo especialmente pacientes de grado severo.

Dr. Angel Golimstok [ Jefe de Sección "Trastornos de Memoria y Conducta". Servicio Neurología. Hospital Italiano de Buenos Aires. ]

Referencias

1. Efficacy of donepezil on behavioral symptoms in patientes with moderate to severe Alzheimer's disease.Gauthier S,Feldman H,Hecker J y col. Int Psychogeritr.2002 Dec;14(4):389-404. 2. The spectrum of behavioral responses to cholinesterase inhibitor therapy in Alzheimer disease.Mega MS, Masterman DM, O"Connor SM y col. Arch.Neurol. 1999Nov;56(11):1388-93. 